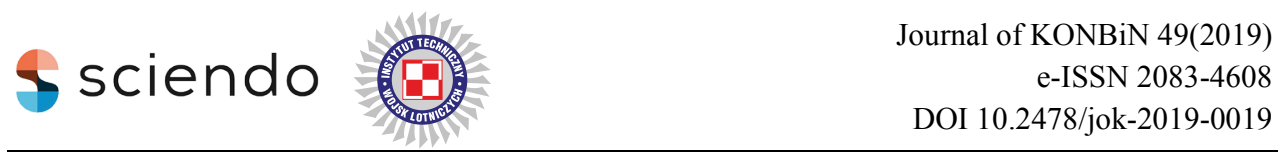

Michał BATSCH

Rzeszów University of Technology (Politechnika Rzeszowska)

\title{
RAPID PROTOTYPING AND TOOTH CONTACT ANALYSIS OF ECCENTRIC CYCLOID GEAR MESH
}

\begin{abstract}
This paper presents a mathematical model and tooth contact analysis of eccentric cycloid gear mesh. Numerical simulations aimed at generating contact pattern for a sample gear pair were performed. Contact pattern was also determined for gear wheels made by additive fabrication from transparent light-activated resin.
\end{abstract}

Keywords: tooth contact analysis, eccentric cycloid gear engagement, rapid prototyping

\section{Introduction}

Today's gears used in the automotive industry should be characterized by noiseless operation, kinematic accuracy and high load-bearing capacity but, at the same time, the smallest possible form factor. Researchers and engineers still attempt to improve gears to increase e.g. their performance and efficiency. This is made possible thanks to the development of materials engineering $[10,11]$ or the application of non-standard meshing geometries [1, 3, 5, 8, 9]. A tooth profile commonly used in gears is the involute profile, in which, in the case of outer meshing, the convex flank of the pinion tooth meshes with the convex flank of the gear tooth. The nature of such gear contact has an adverse effect due to surface tension. An alternative to the involute profile is the Novikov profile, in which tooth profiles have the form of arcs of a circle. The Novikov mesh has greater surface load-bearing capacity than the involute engagement, although it is more susceptible to the axis distance error [8]. Because of such limitations, eccentric-cycloid mesh was designed [15], combining the advantages of involute and Novikov mesh. According to the authors of the solution, the above claim is confirmed by the successful application of the mesh in the transmissions of Kamaz utility vehicles or underground train drives [14]. 
The principle of operation of eccentric-cycloid mesh was presented in [15]. The pinion contains screw teeth with a profile in the form of a circular arc in the frontal section. The gear wheel's screw teeth have the form of an epicycloid equidistant isoline in the frontal section. This mesh design enables high transmission ratios, as it is possible to make a pinion with a small number of teeth or even design it as a single-thread worm [15]. Study [8] presents the mathematical model of teeth which was used to determine the line of contact and perform the FEM strength analysis. The model featured gear wheel tooth surface based on the mesh equation, which means that it constituted an envelope of the family of pinion tooth profile surfaces. Thus, the contact line obtained was a Gaussian coordinate line of the gear wheel tooth surface. The model did not enable an analysis of a gear with gear wheel axis position errors.

Some studies discuss machining such gear teeth on 5-axis CNC machine tools [6].

\section{A mathematical model of eccentric-cycloid mesh}

This study analyses a cylindrical gear with parallel axes and internal and external mesh, composed of two gear wheels with an eccentric-cycloid profile. A pinion with a tooth profile in the form of circular arch mates with a gear wheel with a cycloid tooth profile (fig. 1).

Stationary coordinate system $x_{f}, y_{f}, z_{f}$ connected with the gear case was implemented together with moving coordinate systems fixed to the pinion and the gear, respectively: $x_{1}, y_{1}, z_{1}$ and $x_{2}, y_{2}, z_{2}$. The pinion rotates around axis $z_{1}$ which crosses point $O_{1}$ at angle $\varphi_{1}$ opposite to the trigonometric direction. The wheel rotates around axis $z_{2}$ which crosses point $O_{2}$ at angle $\varphi_{2}$ according to the trigonometric direction (fig. 1). Value $a$ is the distance between wheel centres, and thus coordinate system centres. Pinion tooth surface $\Sigma_{l}$ in coordinate system $x_{l}, y_{l}$, $z_{l}$ is described by position vector $\bar{r}_{1}^{(1)}$. 


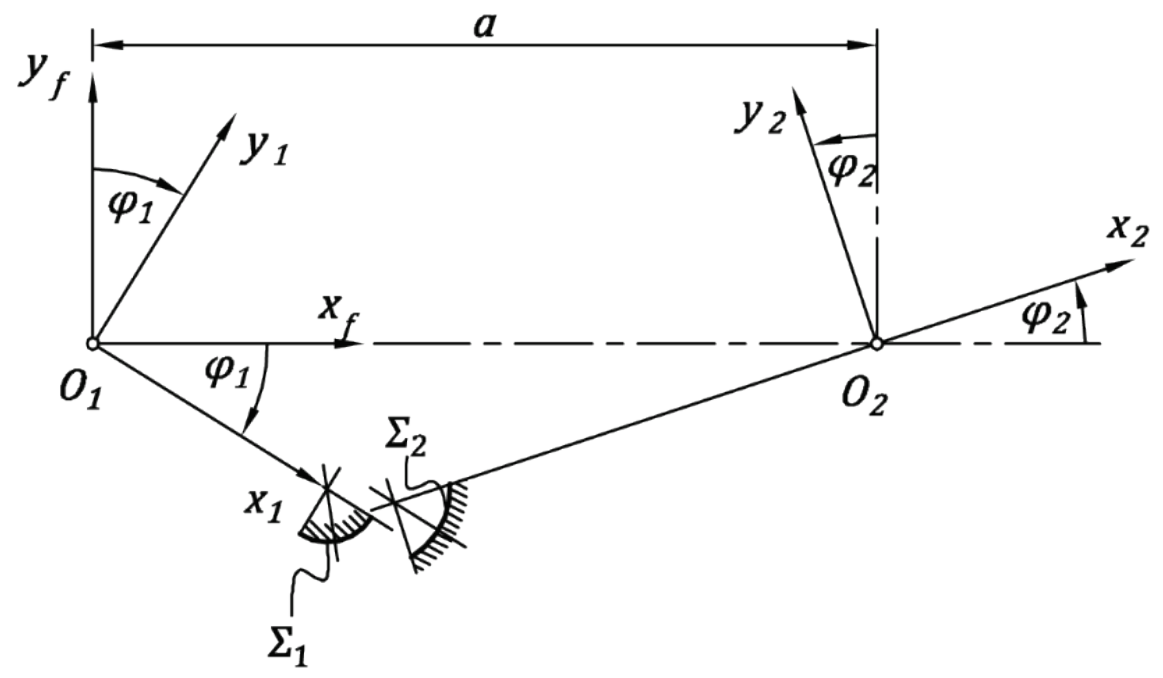

Fig. 1. Coordinate systems applied

Similarly, pinion tooth surface $\Sigma_{2}$ in coordinate system $x_{2}, y_{2}, z_{2}$ is described by position vector $\bar{r}_{2}^{(2)}$. Taking the above into account, according to fig. 1, pinion and wheel surfaces in stationary coordinate system $x_{f}, y_{f}, z_{f}$ will be given by formulae (1) and (2):

$$
\begin{aligned}
& \bar{r}_{1}^{(f)}=M_{f 1} \bar{r}_{1}^{(1)} \\
& \bar{r}_{2}^{(f)}=M_{f 2} \bar{r}_{2}^{(2)}
\end{aligned}
$$

where:

$M_{f 1}$ - homogeneous matrix of transformation from system $l$ to system $f$, $L_{f 2}$ - homogeneous matrix of transformation from system 2 to system $f$.

In order to allow for axis position deviations due to assembly errors, workmanship, elastic deformations of shafts and bearings, the coordinate system connected with the wheel must be shifted along axes $x_{f}, y_{f}, z_{f}$ by values $\Delta a_{x} \Delta a_{y}$ and $\Delta a_{z}$, and then rotated relative to stationary axes $x_{f}$ and $y_{f}$ by angles $\kappa_{x}$ and $\kappa_{y}$ respectively. To this end, additional (auxiliary) coordinate system $x_{h}, y_{h}, z_{h}$ was designed, as shown in fig. 2 . 


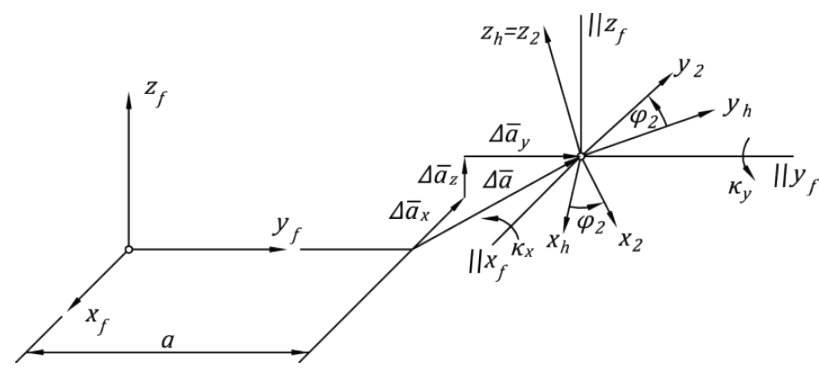

Fig. 2. Additional coordinate system

Allowing for errors, the gear wheel will rotate around a new shifted and skewed axis $z_{h}=z_{2}$. As shown in fig. 2, the matrix of transformation from system 2 to system $f$ is expressed by the formula (3):

$$
M_{f 2}=M_{f h} M_{h 2}
$$

where:

$M_{f h}$ - homogeneous matrix of transformation from system $h$ to system $f$, $M_{h 2}$ - homogeneous matrix of transformation from system 2 to system $h$.

The matrices will have the following form:

$$
\begin{gathered}
M_{f 1}=\left[\begin{array}{cccc}
\cos \varphi_{1} & \sin \varphi_{1} & 0 & 0 \\
-\sin \varphi_{1} & \cos \varphi_{1} & 0 & 0 \\
0 & 0 & 1 & 0 \\
0 & 0 & 0 & 1
\end{array}\right] \\
M_{h 2}=\left[\begin{array}{cccc}
\cos \varphi_{2} & -\sin \varphi_{2} & 0 & 0 \\
\sin \varphi_{2} & \cos \varphi_{2} & 0 & 0 \\
0 & 0 & 1 & 0 \\
0 & 0 & 0 & 1
\end{array}\right] \\
M_{f h}=\left[\begin{array}{cccc}
\cos \kappa_{y} & \sin \kappa_{x} \sin \kappa_{y} & \cos \kappa_{x} \sin \kappa_{y} & a+\Delta a_{x} \\
0 & \cos \kappa_{y} & -\sin \kappa_{x} & \Delta a_{y} \\
\sin \kappa_{y} & \sin \kappa_{x} \cos \kappa_{y} & \cos \kappa_{x} \cos \kappa_{y} & \Delta a_{z} \\
0 & 0 & 0 & 1
\end{array}\right]
\end{gathered}
$$

Moreover, a description of the surfaces requires homogeneous vector representations.

Assuming that $\bar{n}_{1}^{(1)}$ and $\bar{n}_{2}^{(2)}$ are versors normal to tooth surfaces of the pinion and the gear wheel respectively in systems $l$ and 2 , normal versors in system $f$ will be provided by the following relationships: 


$$
\begin{aligned}
& \bar{n}_{1}^{(f)}=L_{f 1} \bar{n}_{1}^{(1)} \\
& \bar{n}_{2}^{(f)}=L_{f 2} \bar{n}_{2}^{(2)}
\end{aligned}
$$

where:

$L_{f 1}$ - matrix of transformation from system 1 to $f$, $L_{f 2}$ - matrix of transformation from system 2 to $f$.

The matrices in formulae (7) and (8) are obtained by removing the last line and the last column of homogeneous transformation matrix (4) $\div(6)$.

\section{Tooth flank parametric equations}

Figure 3 presents a frontal section of mating gear wheels for zero wheel rotation angles.
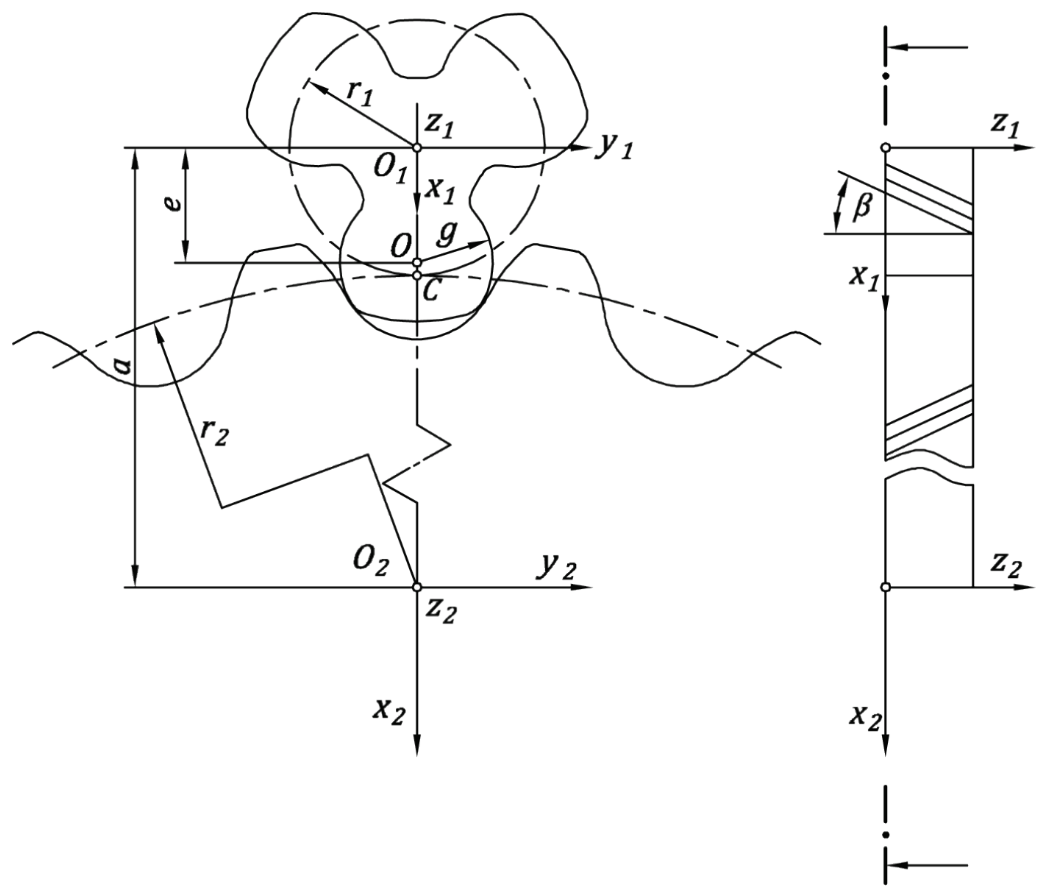

Fig. 3. Frontal section of a gear with eccentric-cycloid mesh, where: $\mathrm{r}_{1}$ - pinion pitch radius, $\mathrm{r}_{2}$ - gear wheel pitch radius, $\mathrm{g}-$ pinion tooth profile radius (equidistant isoline displacement distance), a - distance between gear wheel axes, $\mathrm{C}$ - mesh centre, $\mathrm{O}$ - pinion tooth profile centre, $\beta$ - tooth line inclination angle on pitch diameter. 
A pinion of pitch radius $r_{l}$ mates with a gear wheel of pitch radius $r_{2}$. $C$ is the mesh centre being the point of contact of pitch circles. $O$ is the centre of the tooth profile of the pinion with radius $g$. Its position is defined by eccentric $e$. The pinion profile is described by equation system (9)

$$
\left\{\begin{array}{l}
x_{1}=g \cos \eta_{1}+e \\
y_{1}=g \sin \eta_{1}
\end{array}\right.
$$

where: $\eta_{1}$ is a parameter of the circle.

The gear wheel's tooth outline is the internal equidistant of the epicycloid. Assuming that $g=v \cdot r_{1}$, where $v$ is the equidistant isoline's shift ratio (for an internal equidistant $v<0$ ) and $\lambda$ is the tooth depth ratio, the gear wheel tooth profile is given by coordinate system $(10)$

$$
\left\{\begin{array}{l}
x_{2}=\frac{z_{1} m_{t}}{2}\left(\left(\frac{z_{2}}{z_{1}}+1\right) \cos \eta_{2}-\lambda \cos \left(\left(\frac{z_{2}}{z_{1}}+1\right) \eta_{2}\right)+v \frac{\cos \eta_{2}-\lambda \cos \left(\left(\frac{z_{2}}{z_{1}}+1\right) \eta_{2}\right)}{\sqrt{1-2 \lambda \cos \left(\frac{z_{2}}{z_{1}} \eta_{2}\right)+\lambda^{2}}}\right) \\
y_{2}=\frac{z_{1} m_{t}}{2}\left(\left(\frac{z_{2}}{z_{1}}+1\right) \sin \eta_{2}-\lambda \sin \left(\left(\frac{z_{2}}{z_{1}}+1\right) \eta_{2}\right)+v \frac{\sin \eta_{2}-\lambda \sin \left(\left(\frac{z_{2}}{z_{1}}+1\right) \eta_{2}\right)}{\sqrt{1-2 \lambda \cos \left(\frac{z_{2}}{z_{1}} \eta_{2}\right)+\lambda^{2}}}\right)
\end{array}\right.
$$

where $\eta_{2}$ is a parameter of the epicycloid.

Parametric equation of the pinion tooth flank (11) was generated by simultaneous rotation around axis $z_{1}$ by angle $\zeta_{1}$ according to the trigonometric direction and translation of circular arch (9) along the positive direction of axis $z_{1}$ o $\zeta_{1} r_{1} \operatorname{ctg} \beta$ :

$$
\bar{r}_{1}^{(1)}=\left[\begin{array}{cccc}
\cos \xi_{1} & \sin \xi_{1} & 0 & 0 \\
-\sin \xi_{1} & \cos \xi_{1} & 0 & 0 \\
0 & 0 & 0 & \zeta_{1} r_{1} \operatorname{ctg} \beta \\
0 & 0 & 0 & 1
\end{array}\right] \cdot\left[\begin{array}{c}
x_{1} \\
y_{1} \\
0 \\
1
\end{array}\right]
$$

Parametric equation of the gear wheel tooth flank (12) was obtained in a similar way, with the exception that the rotation of tooth profile (10) was around axis $z_{2}$ by 
angle $\zeta_{2}$ opposite to the trigonometric direction, and the translation was performed along the positive direction of axis $z_{2} \mathrm{o} \zeta_{2} r_{2} c t g \beta$ :

$$
\bar{r}_{2}^{(2)}=\left[\begin{array}{cccc}
-1 & 0 & 0 & 0 \\
0 & -1 & 0 & 0 \\
0 & 0 & 1 & 0 \\
0 & 0 & 0 & 1
\end{array}\right] \cdot\left[\begin{array}{cccc}
\cos \xi_{2} & -\sin \xi_{2} & 0 & 0 \\
\sin \xi_{2} & \cos \xi_{2} & 0 & 0 \\
0 & 0 & 0 & \zeta_{2} r_{2} \operatorname{ctg} \beta \\
0 & 0 & 0 & 1
\end{array}\right] \cdot\left[\begin{array}{c}
x_{2} \\
y_{2} \\
0 \\
1
\end{array}\right] .
$$

Moreover, the surface of the gear wheel tooth was rotated by $180^{\circ}$ around axis $z_{2}$ so as to occupy positions presented in fig. 3 .

Versors normal to the flank of the pinion and the gear wheel respectively in coordinate systems 1 and 2 are expressed by formulae (13) and (14):

$$
\begin{aligned}
& \bar{n}_{1}^{(1)}=\frac{\frac{\partial r_{1}^{(1)}}{\partial \zeta_{1}} \times \frac{\partial r_{1}^{(1)}}{\partial \eta_{1}}}{\left|\frac{\partial r_{1}^{(1)}}{\partial \zeta_{1}} \times \frac{\partial r_{1}^{(1)}}{\partial \eta_{1}}\right|} \\
& \bar{n}_{2}^{(2)}=\frac{\frac{\partial r_{2}^{(2)}}{\partial \eta_{2}} \times \frac{\partial r_{2}^{(2)}}{\partial \zeta_{2}}}{\left|\frac{\partial r_{2}^{(2)}}{\partial \eta_{2}} \times \frac{\partial r_{2}^{(2)}}{\partial \zeta_{2}}\right|}
\end{aligned}
$$

\section{Tooth contact analysis}

Tooth contact pattern was determined using a numerical method in which it constitutes a set of points on the tooth surface, for which the distance between tooth flanks measured along a normal versor is less or equal to the set value [7]. Figure 4 illustrates a method for determining the distance between mating tooth flanks on the basis of position vectors and distance vector. 


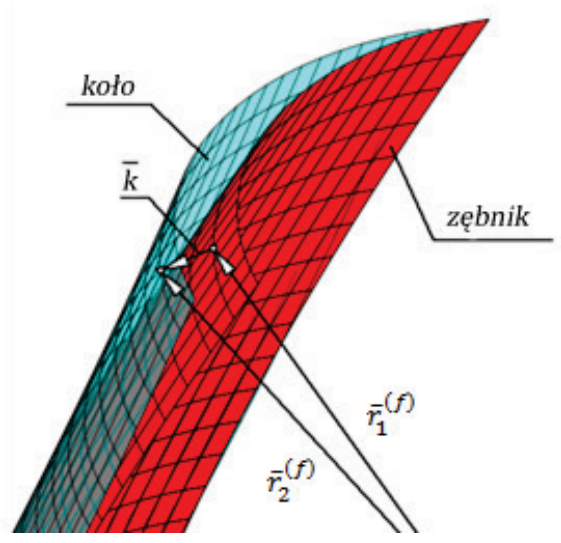

Fig. 4. Determining the distance between mating tooth flanks

The above drawing provides the basis for vector equation (15)

$$
\bar{r}_{1}^{(f)}\left(\eta_{1}, \zeta_{1}\right)+\bar{k}\left(\eta_{1}, \zeta_{1}\right)=\bar{r}_{2}^{(f)}\left(\eta_{2}, \zeta_{2}\right)
$$

where $\bar{k}$ is the distance vector determined by relationship (16)

$$
\bar{k}=k \bar{n}_{1}^{(f)}
$$

Formula (16) is the product of the measure of length vector $k$ and normal versor (13). In order to determine the distance of vector $\bar{k}$ with set parameters $\eta_{1}$ and $\zeta_{1}$, a system of three equations with unknown quantities $k, \eta_{2}$ and $\zeta_{2}(17)$, expressed by a vector equation (15)

$$
\left\{\begin{array}{l}
r_{x 1}^{(f)}\left(\theta_{1}, \zeta_{1}\right)+k n_{x 1}^{(f)}\left(\theta_{1}, \zeta_{1}\right)=r_{x 2}^{(f)}\left(\theta_{2}, \zeta_{2}\right) \\
r_{y 1}^{(f)}\left(\theta_{1}, \zeta_{1}\right)+k n_{y 1}^{(f)}\left(\theta_{1}, \zeta_{1}\right)=r_{y 2}^{(f)}\left(\theta_{2}, \zeta_{2}\right) \\
r_{z 1}^{(f)}\left(\theta_{1}, \zeta_{1}\right)+k n_{z 1}^{(f)}\left(\theta_{1}, \zeta_{1}\right)=r_{z 2}^{(f)}\left(\theta_{2}, \zeta_{2}\right)
\end{array}\right.
$$

The contact area of the gear wheels is obtained in graph $r_{M 1}\left(b_{M 1}\right)$ by combining points for which $k$ is lower than the thickness of marking compound in the gear marking method. The values $r_{M 1}$ and $b_{M 1}$ are provided by formulae (18)

$$
r_{M 1}=\sqrt{r_{x 1}^{2}+r_{y 1}^{2}}, b_{M 1}=r_{z 1}
$$


The analyses were performed for gears of parameters listed in table 1.

Table 1

Data of the analysed gear

\begin{tabular}{|c|c|c|}
\hline & Pinion & Wheel \\
\hline Normal module $[\mathrm{mm}]$ & \multicolumn{2}{|c|}{$m_{n}=8$} \\
\hline Number of teeth [-] & $z_{1}=3$ & $z_{2}=18$ \\
\hline Tooth contact ratio [-] & \multicolumn{2}{|c|}{$\varepsilon_{\beta}=1,1286$} \\
\hline Crown width [mm] & \multicolumn{2}{|c|}{$b=50$} \\
\hline Tooth line inclination angle $\left[^{\circ}\right]$ & \multicolumn{2}{|c|}{$\beta=34,5603$} \\
\hline Equidistant isoline shift ratio [-] & \multicolumn{2}{|c|}{$v=-0,6$} \\
\hline Tooth depth ratio [-] & \multicolumn{2}{|c|}{$\lambda=0,9$} \\
\hline Pitch diameter $[\mathrm{mm}]$ & $d_{1}=29,1426$ & $d_{2}=174,8571$ \\
\hline Tip diameter [mm] & $d_{a 1}=39,7143$ & $d_{a 2}=184$ \\
\hline Root diameter $[\mathrm{m}]$ & $d_{f 1}=16$ & $d_{f 2}=160,2857$ \\
\hline
\end{tabular}

Contact patterns were determined for $k=0.05 \mathrm{~mm}$. Figure 5 shows the effect of equidistant isoline shift ratio on the contact pattern.

a)

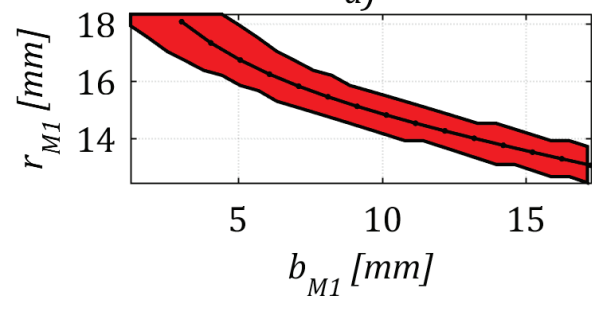

c)

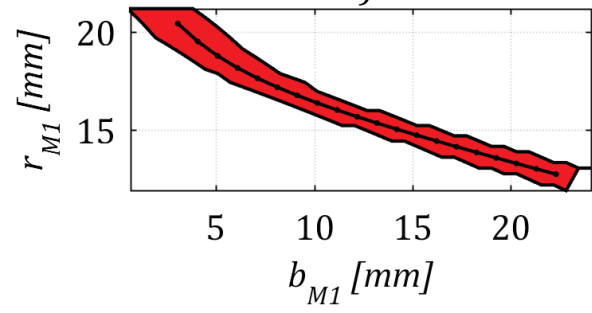

b)

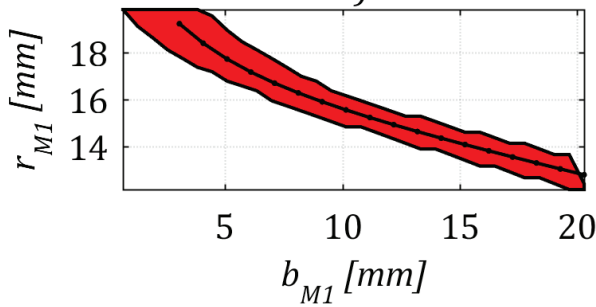

d)

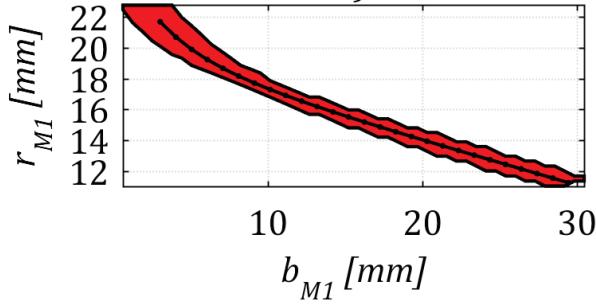

Fig. 5. The effect of equidistant isoline ratio on the contact pattern: a) $v=-0.5$, b) $v=-0.6$, c) $v=-0.7$, d) $v=-0.8$ 
The contact area is marked in red. The black line shows a theoretical tooth contact line. The lower the $v$ ratio, the smaller the contact area. In all of the above cases the contact pattern is wider at the pinion tooth tip and becomes narrower as it approaches the root. Figure 6 shows the effect of tooth depth ratio on the contact pattern.
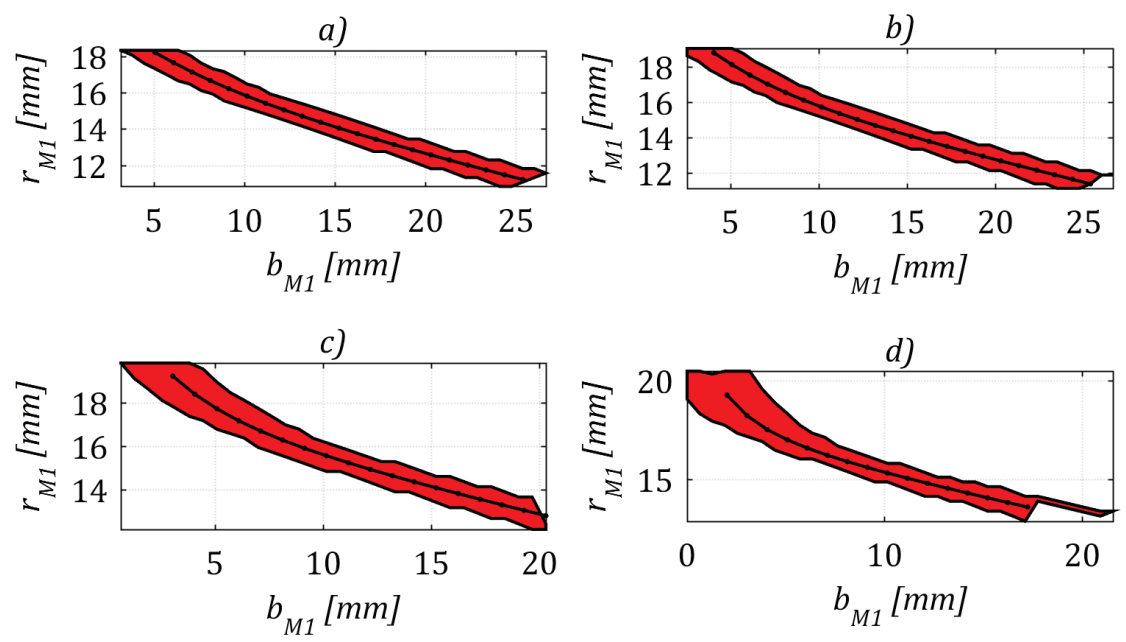

Fig. 6. The effect of the tooth depth ratio on the contact pattern: a) $\lambda=0.8, b) \lambda=0.85$, c) $\lambda=0.9$, d) $\lambda=0.95$

Along with the rise in ratio $\lambda$ the contact pattern area increases, becoming wider at the pinion tooth tip.

\section{An experimental method of determining instantaneous contact pattern}

An experimental method of determining instantaneous contact pattern in a gear was designed in the Machine Design Department at Rzeszów University of Technology $[12,13]$. The method involves fabricating wheels of the prototype gear from a transparent material. The marking compound used is a liquid (e.g. water, water with detergent, oil) whose meniscus corresponds to the equidistant contour line between teeth, and which is used to ascertain the instantaneous contact pattern. The method, coupled with the contact pattern assessment approach, was successfully used for the verification of the mathematical model of covexo-concave Novikov mesh [2]. 
Figure 7 shows a test rig for determining instantaneous contact pattern. Its components were fabricated by means of an additive technique (PolyJet) from transparent light-activated resin. Pinion 1 mates with gear wheel 2, in which one tooth space is shaped as a thin-walled component, allowing us to observe the contact pattern. Meshing data are listed in table 1.

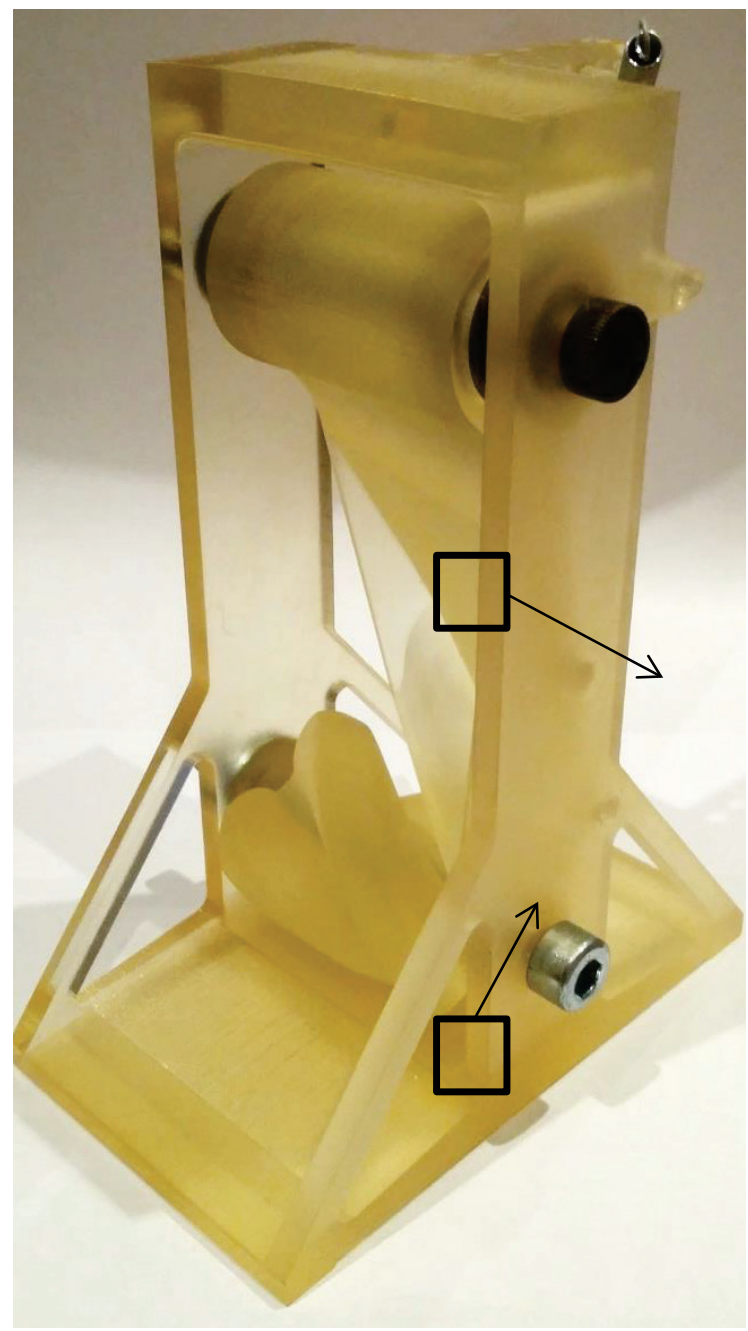

Fig. 7. A test rig for studying instantaneous contact pattern in an eccentric-cycloid mesh

The size of the contact pattern may be expressed as percentage share of the tooth's surface. To determine the share, an image-processing algorithm [2] was used, the consecutive steps of which are shown in fig. 8. 


\section{a) Contact pattern image}

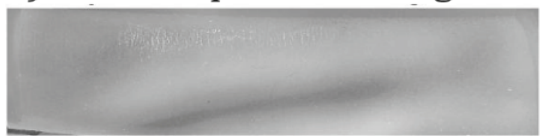

c) Noise reduction

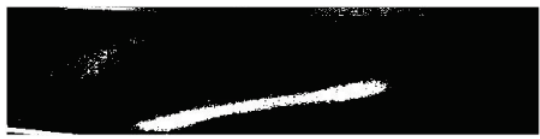

e) Noise reduction

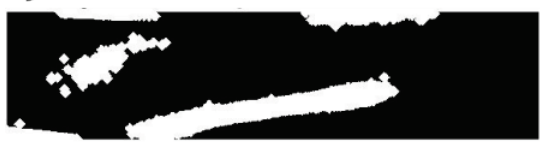

g) Erosion

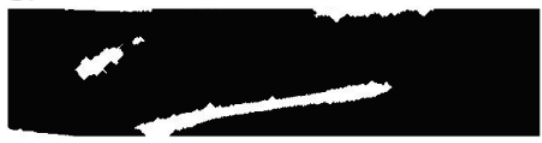

b)Threshholding

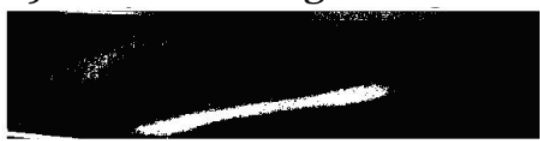

d) Dilation

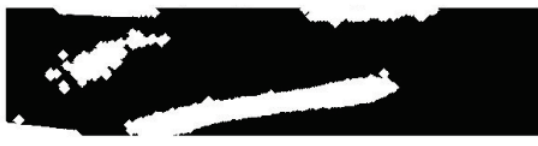

f) Hole filling

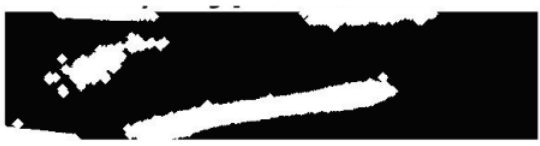

h) Noise reduction

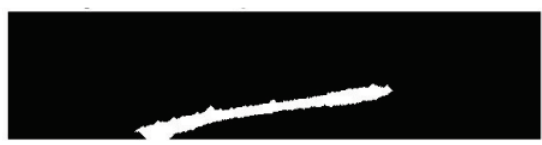

Fig. 8. Consecutive steps of the contact pattern image processing algorithm

The image was suitably processed prior to the initiation of the algorithm. Its contrast was increased and any shadows present were removed. The algorithm starts with loading a monochromatic image of tooth surface (fig. 8a). Next, the image is transformed into a binary set of points and inverted (fig. 8b). Then, the image is dilated (fig. 8d), filled in (fig. 8f) and eroded (fig. 8g). If required, noise is removed between subsequent steps. The final step of the algorithm includes counting the number of white pixels and calculating the ratio of white pixels to total image. Such ratio corresponds to percentage contact area. The percentage share of the contact area determined using the experimental method is shown in fig. 8 and equals $4.63 \%$. The percentage share of the contact area determined numerically (fig. $5 b$ ) equals $4.40 \%$. The differences are slight and may arise from inaccurate fabrication of surfaces by means of rapid prototyping techniques as well as a measuring method error. 


\section{Summary and conclusions}

In this paper, a mathematical model of tooth contact analysis in an eccentric cycloid gear mesh was presented. Moreover, the study proved the usefulness of the model in tooth contact analysis and modelling such tooth design. On the basis of the analyses performed in the study it was concluded that:

- an increase in the tooth depth ratio allows us to extend tooth contact area, thus reducing contact stress,

- the use of a lower value of equidistant isoline shift ratio causes a decrease in the contact area and increases surface tension,

- in operation, similarly to a convexo-concave mesh, e.g. Novikov-type mesh, the contact pattern moves along the tooth line,

- the experimental method of determining instantaneous contact pattern using transparent models may be successfully used to evaluate contact pattern in a gear transmission.

\section{References}

1. Batsch M., Homik W., Markowski T.: Cylindrical Gears with Increased Contact Area - Proposal of Application in Watercrafts Power Transmission Systems. Solid State Phenomena, Vol. 236, 2015.

2. Batsch M., Markowski T., Legutko S. et al.: Measurement and mathematical model of convexo-concave Novikov gear mesh, Measurement, Vol. 125, 2018.

3. Batsch M., Markowski T.: Comparative fatigue testing of gears with involute and convexo-concave teeth profiles. Advances in Manufacturing Science and Technology, Vol. 40, No. 2, 2016.

4. Batsch M.: The Method of Axes Distance Error Compensation in Convexo-Concave Novikov Gears. Advances in Manufacturing Science and Technology, Vol. 39, No. 4, 2015.

5. Dyson A., Evans H.P., Snidle W., Wildhaber-Novikov circular arc gears: Geometry and Kinematics. Proceedings of The Royal Society A Mathematical Physical and Engineering Sciences, Vol. 403, No. 1825, 1986.

6. Kamchatnyi S.A., Kuznetsov V.M., Skovorodin A.V. et al.: Mathematical simulation of the formbuilding of the conic driving gear details with EC-gearing. Vestn. Tomsk. Gos. Univ. Mat. Mekh., Vol. 2, No. 28, 2014.

7. Kawalec A., Wiktor J.: Simulation of generation and tooth contact analysis of helical gears with crowned flanks. Proceedings of the Institution of Mechanical Engineers, Part B: Journal of Engineering Manufacture, Vol. 222, No. 9, 2008. 
8. Li X., Li C., Chen B. et al.: Design and investigation of a cycloid helical gear drive. Journal of Mechanical Science and Technology, Vol. 31, No. 9, 2017.

9. Litvin F.L., Fuentes A., Gonzalez-Perez I. et al.: New version of Novikov-Wildhaber helical gears: computerized design, simulation of meshing and stress analysis. Computer Methods in Applied Mechanics and Engineering, Vol. 191, No. 49-50, 2002.

10. Rokicki P., Dychtoń K., Drajewicz M. et al.: Thermal analysis of low alloyed steel for heavily-loaded aircraft engine transmission gears. Advances in Manufacturing Science and Technology, Vol. 39, No. 3, 2015.

11. Rokicki P., Dychtoń K., Nowotnik A. et al.: Determination of heat treatment parameters for heavily-loaded aircraft engine components. Journal of KONES, Vol. 20, No. 2, 2013.

12. Sobolak M., Budzik G.: Experimental method of tooth contact analysis (TCA) with rapid prototyping (RP) use, Rapid Prototyping Journal, Vol. 14, No. 4, 2008.

13. Sobolak, M., Experimental method of tooth contact analysis (TCA) in toothed gear, Archives of Mechanical Technology and Automation, Vol. 27, No. 2, 2007.

14. Stanovskoy V.V., Kazakyavichyus S.M., Kuznetsov B.M.: Razrabotka i vniedreniye resursosberegayushchey techniki novogo pokoleniya na osnove excentricovocicloidalogo (EC) zacepleniya, Innovatsii, Vol. 12, No. 182, 2013.

15. Stanovskoy V.V., Kazakyavichyus S.M., Remneva T.A. et al.: US Patent No. 8,789,437 B2, 2014. 